\title{
Application values of clinical nursing pathway in patients with acute cerebral hemorrhage
}

\author{
WEIHUA LI ${ }^{*}$, JIANMEI GAO $^{2 *}$, SHUFANG WEI ${ }^{3}$ and DONGHAI WANG ${ }^{4}$ \\ ${ }^{1}$ Operating Room, Qilu Hospital of Shandong University, Jinan, Shandong 250012; ${ }^{2}$ Operating Room, \\ Shandong Chest Hospital, Jinan, Shandong 250013; ${ }^{3}$ Comprehensive Ward; ${ }^{4}$ Department of Neurological Surgery, \\ Qilu Hospital of Shandong University, Jinan, Shandong 250012, P.R. China
}

Received October 26, 2015; Accepted December 3, 2015

DOI: $10.3892 /$ etm.2015.2909

\begin{abstract}
Acute cerebral hemorrhage accounts for approximately $25 \%$ of strokes for elderly patients. Consequently, treatments to improve prognosis should be identified. The aim of the present study was to examine the clinical values of the application of clinical nursing pathway for patients with acute cerebral hemorrhage. Between January 2013 and January 2015, 92 patients diagnosed with acute intracerebral hemorrhage were enrolled in the study based on the guidelines recommended for providing appropriate surgical or conservative treatment and the sequence of admission. The 92 patients were randomly divided into the control and observation groups. Patients in the control group underwent routine nursing mode prior to and after admission, and underwent clinical nursing path model (hierarchical partitioning prior to admission to hospital plus general professional program of nursing in hospital) was applied to the observation group. Barthel index scores for the observation group were significantly higher than that of the control group. The length of hospital stay for patients in the observation group was significantly lower while the average score for patients' satisfaction on nursing care while in hospital was significantly higher than that of the control group, with statistically significant differences $(\mathrm{P}<0.05)$. The incidence of complications such as fever, infection, bedsore, gastrointestinal function, electrolyte disturbances, and malnutrition, in the observation group was significantly lower, with statistically significant differences $(\mathrm{P}<0.05)$. The functional independence measure (FIM) and Fugl-Meyer scores after 6 months for the observation group were significantly higher, with statistically
\end{abstract}

Correspondence to: Dr Donghai Wang, Department of Neurological Surgery, Qilu Hospital of Shandong University, 107 Cultural West Road, Jinan, Shandong 250012, P.R. China

E-mail: wrkne204@163.com

*Contributed equally

Key words: clinical nursing pathway, acute cerebral hemorrhage, Barthel index, nursing job satisfaction scale, functional independence measure score, Fugl-Meyer score significant differences $(\mathrm{P}<0.05)$. In conclusion, application of the clinical nursing pathway for patients with acute cerebral hemorrhage significantly improved the clinical effects and nursing satisfaction, reduced adverse reactions, and had a greater clinical application value.

\section{Introduction}

Cerebral hemorrhage is a non-traumatic and spontaneous hemorrhage of cerebral parenchyma, caused by blood vessels rupturing owing to hypertensive arteriolar sclerosis. Internal capsule arterial hemorrhage is the primary site of predilection in clinic. Cerebral hemorrhage has an acute onset, rapid development, early onset of hemiplegia and disturbance of consciousness. Morbidity and mortality are high, making it one of the most lethal diseases (1). Acute cerebral hemorrhage has a high incidence in elderly patients, accounting for $\sim 25 \%$ of all strokes (2).

Clinical manifestations following acute cerebral hemorrhage depend on the speed and amount of hemorrhage. Clinical manifestations such as brain edema, intracranial hypertension, high fever, cerebral hernia, limb and speech disorder, breathing and heartbeat inhibition, shock and even death have been reported (3). Cerebral hemorrhage survivors may suffer from a number of complications, which may markedly affect both patients and their families. Cerebral hemorrhage has a high disability rate and even higher mortality rate (4). Good clinical practice, proper care, medication and rehabilitation can greatly increase the treatment rate, and improve the prognosis. Clinical nursing path is an interdisciplinary, in-depth integrated, procedural modern nursing mode, with an emphasis on hierarchical partitioning prior to admission as well as a general professional program of nursing in hospitals, to improve the comprehensive caring nursing service for patients (5).

In the present study, the medical values of clinical nursing pathway for patients with acute cerebral hemorrhage was examined to reveal and provide novel ideas and experiences for developing new methods in nursing.

\section{Materials and methods}

General materials. Signed informed consent forms were obtained from the patients and their families for 
participation in the present study. The study was approved by the Ethics Committee of Qilu Hospital of Shandong University (Shandong, China). A total of 92 patients diagnosed with acute intracerebral hemorrhage were enrolled in the study between January 2013 and January 2015, at the Qilu Hospital of Shandong University. All 92 patients met the diagnostic criteria of acute cerebral hemorrhage (6), which included sudden clinical symptoms, such as dizziness, headache, poor body condition and speech, falls and coma, and confirmation of presence of fresh and high-density shadow by emergency computed tomography (CT).

The 92 cases adhered to the inclusion criteria of being between 18 and 70 years of age, and meeting the diagnostic criteria of acute cerebral hemorrhage. Additionally, it was the first time that the patients presented to the hospital for cerebral hemorrhage.

Patients with traumatic intracerebral hemorrhage, hemorrhage hemophilia, cerebral vascular malformation, autoimmune cerebral hemorrhage, and tumor hemorrhage were excluded. Cases with severe coagulation dysfunction, heart, liver, kidney and other organ dysfunction, large bleeding volume, and poor prognosis were excluded. Parturient females and patients refusing to participate in the current study were also excluded.

For this study, the patients were included based on the guidelines recommended for the provision of appropriate surgical or conservative treatment and according to the sequence of admission. Cases were randomly divided into the control group ( $n=43$ cases) and observation group ( $n=49$ cases). The study included 29 males and 14 females in the control group, aged 42-69 years, with an average age of $(56.5 \pm 8.1)$ years. The subjects underwent $30 \mathrm{~min}$ to $8 \mathrm{~h}$ of bleeding (average of $3.4 \pm 1.2 \mathrm{~h}$ ). The bleeding volume for patients in the control group ranged $3-12 \mathrm{ml}$ with an average of $5.7 \pm 1.3 \mathrm{ml}$. There were 17 cases of lobar hemorrhage, 6 cases of cerebellum, 10 cases of thalamus, and 10 cases of basal ganglia, as well as 21 cases of combined hypertension, 5 cases of diabetes, and 7 cases of coronary heart disease in the control group.

The observation group comprised 34 males and 15 females, aged 38-67 years, with an average age of 57.4 \pm 10.2 years. The bleeding time for patients in the observation group was $45 \mathrm{~min}$ to $9.5 \mathrm{~h}$ (average $3.7 \pm 1.1 \mathrm{~h}$ ). For the bleeding sites, there were 19 cases of lobar hemorrhage, 8 cases of cerebellum, 13 cases of thalamus, and 19 cases of basal ganglia, as well as 23 cases of combined hypertension, 6 cases of diabetes, and 9 cases with coronary heart disease. No statistically significant differences were identified following a comparison of gender, age, bleeding time, bleeding volume, bleeding site and the combined disease of the two groups $(\mathrm{P}>0.05)$.

Test method. Patients in the control group underwent routine and conventional nursing mode prior to and after admission to the hospital. The patients were admitted to the hospital primarily through emergency, outpatient registration or emergency green channel, and physicians who diagnosed the disease. To determine the severity of the disease, the patients were examined with emergency CT on admission. To prevent complications, including indwelling central venous access, nasogastric enteral nutrition support, catheter, continuous monitoring of oxygen, vital signs, physical cooling, alcohol sponge bath and antipyretic drug treatment were considered. The nurses administered medications and prepared patients for surgery as per the physician's instructions. The situation was explained to the family, who were informed that the conditions may change.

The observation group received the clinical nursing path model $(7,8)$. This model included: i) Hierarchical partitioning prior to admission to the hospital where patients from the green channel, received a rapid diagnosis followed by an accurate examination, application of vital signs support and emergency medication, and rapid transfer to the relevant specialists, ii) general professional program of nursing in hospital. On the day of admission, the nursing team combined the actual situation of the patients with the domestic and international standards for the development of nursing path and according to the encountered actual operation problems added and modified the nursing content. The patients were guided through their medication and care schedules, and their diets were monitored. The nursing team evaluated the general condition of the patients, and preventive measures for seizures and other adverse events were conducted using emergency equipment and proper medication.

At 2-7 days after admission, the shift nurse assessed the patient's condition and limbs, and examined the condition of skin, pupils, as well as whether the patient had a choking cough. The nurse-in-charge, the responsible nurse and head nurse engaged in ward management together and verified the proper implementation of the nursing plan and clinical treatment process. Any problem that arose was promptly corrected by the nursing team entrusted to care for the patients and family. The patients received continuous assessment and were provided information. The nursing team communicated information regarding cerebral hemorrhage with patients and their families, and depending on the situation, psychological interventions were implemented to reduce their anxiety. In the case of a coma, health education and psychological nursing were provided to the family members. At 8-14 days after admission, the patients were guided and assisted with early rehabilitation education and training.

Following discharge from the hospital, the nursing team assessed the achievements of the nursing path plan to objectively record the deviations. The patients were provided diet guidance and relevant advice on the importance of drinking an adequate quantity of water. Risks associated with constipation as well as prevention thereof were explained in detail. Pertinent information on how to undergo functional recovery training, and how to approach work and rest was distributed. Patients were informed of any possible side effects and adverse effect associated with their medications and patients were assisted with the referral notes. Through all the sessions with patients, the nurses emphasized the importance of positive thinking and an optimistic attitude.

Observation index. A comparative study was performed on the Barthel index scores, the average scores of patient satisfaction with nursing care, hospitalization time, and the incidence of complications such as fever, infection, bedsore, gastrointestinal function and electrolyte disturbances, malnutrition prior to and after nursing in the two groups. Functional 
Table I. Comparison of Barthel index score and average total score of patient satisfaction following nursing care and length of hospital stay between the two groups.

\begin{tabular}{lcccc}
\hline Group & $\begin{array}{c}\text { Barthel index } \\
\text { score prior to nursing }\end{array}$ & $\begin{array}{c}\text { Barthel index } \\
\text { score after nursing }\end{array}$ & $\begin{array}{c}\text { Average total score of patient } \\
\text { satisfaction with nursing care }\end{array}$ & $\begin{array}{c}\text { Length of hospital stay, } \\
\text { days }\end{array}$ \\
\hline Control & $41.5 \pm 8.2$ & $56.7 \pm 9.4$ & $29.7 \pm 4.3$ & $15.3 \pm 2.1$ \\
Observation & $40.7 \pm 7.6$ & $72.8 \pm 8.8$ & $37.8 \pm 3.5$ & $10.9 \pm 2.3$ \\
t-test & 0.527 & 5.402 & 4.636 & 3.758 \\
P-value & 0.941 & 0.019 & 0.027 & 0.036 \\
\hline
\end{tabular}

Table II. Comparison of the incidence of complications in the two groups [cases (\%)].

\begin{tabular}{|c|c|c|c|c|c|c|c|}
\hline Group & Cases & High fever & Infected & Bedsore & $\begin{array}{l}\text { Gastrointestinal function } \\
\text { and electrolyte disorders }\end{array}$ & Malnutrition & $\begin{array}{c}\text { Incidence } \\
\text { of complications }\end{array}$ \\
\hline Control group & 43 & 3 & 2 & 3 & 1 & 1 & $10(23.3 \%)$ \\
\hline Observation group & 49 & 1 & 0 & 1 & 2 & 0 & $4(8.2 \%)$ \\
\hline$\chi^{2}$ test & & & & & & & 4.043 \\
\hline P-value & & & & & & & 0.044 \\
\hline
\end{tabular}

Table III. Comparison of FIM and Fugl-Meyer scores between the two groups after 6 months follow up.

\begin{tabular}{lcccc}
\hline Group & $\begin{array}{c}\text { FIM score } \\
\text { prior to nursing }\end{array}$ & $\begin{array}{c}\text { FIM score after } \\
\text { follow up }\end{array}$ & $\begin{array}{c}\text { Fugl-Meyer score } \\
\text { prior to nursing }\end{array}$ & $\begin{array}{c}\text { Fugl-Meyer score } \\
\text { after follow up }\end{array}$ \\
\hline Control & $35.7 \pm 6.5$ & $56.9 \pm 10.2$ & $42.5 \pm 7.7$ & $70.6 \pm 12.4$ \\
Observation & $34.6 \pm 5.8$ & $72.4 \pm 8.9$ & $41.9 \pm 6.9$ & $86.9 \pm 11.3$ \\
t-test & 0.657 & 6.417 & 0.928 & 6.398 \\
P-value & 0.416 & $<0.001$ & 0.307 & $<0.001$ \\
\hline
\end{tabular}

FIM, functional independence measure.

independence measure (FIM) and Fugl Meyer scores were analyzed 6 months following admission.

The Barthel index score included: i) meal (10 and 5 points), ii) bed-a wheelchair (15, 10 and 5 points), iii) modified (5 points), iv) ability to enter and exit bathroom facilities (10 and 5 points), v) bath (5 points), vi) walking (15, 10 and 5 points), vii) ability to climb up and down stairs (10 and 5 points), viii) wear clothes (10 and 5 points), ix) stool control (10 and 5 points), and $\mathrm{x}$ ) urinary control (10 and 5 points). Higher scores were associated with a better prognosis.

Hospitalized patient satisfaction with nursing care scale included: i) The environment of the ward, ii) the nurse's physical appearance, iii) quality and safety of the technology used, iv) timely service, v) health education, vi) humanistic values exercised in the care-giving process, vii) service attitude, and viii) education in and out of the hospital. To assess the eight aspects of patient satisfaction, there were 1-4 questions for each aspect. For each question the answer was classified into 5 grades: i) Very dissatisfied (1 point), ii) not satisfied ( 2 points), iii) just so-so ( 3 points), iv) satisfied ( 4 points), and v) very satisfied (5 points).
The FIM scale included: i) Self-care activities (feeding, grooming modification, bathing, wearing vests of the torso, wearing vests and use of bathroom facilities), ii) sphincter control (management of urination and defecation management), iii) transfer (transfer between bed and chair, transfer to the bathroom facilities, transfer to the bath or shower), iv) travel (walking or with a wheelchair, climbing up and down stairs), v) communication (understanding and expression), and vi) social cognition (social interaction, problem solving and memory). Higher scores were associated with a better prognosis.

The Fugl-Meyer motor function score included: i) The upper seat (reflex activity, flexor synergy sports, extensor synergy movement, activity of CO movement, activity out of synergy, hyperreflexia, wrist stability, elbow extension, shoulder flexion 30 degrees, fingers, collaborative ability and speed) and ii) supine position of lower limb (reflex activity, cooperative motion flexor, extensor synergy movement, activity of $\mathrm{CO}$ movement, activity out of synergy, hyperreflexia, collaborative ability and speed). Total scores of FMA were grade I or severe movement disorders ( $<50$ points), grade II or obvious 
dyskinesia (50-84 points), grade III or moderately movement disorders (85-95 points), and grade IV or mild dyskinesia (96-99 points).

Statistical analysis. The SPSS19.0 statistical software (SPSS, Inc., Chicago, IL, USA) package was used for data entry and analysis. Measurement data were presented as mean \pm standard deviation. Comparison between groups was performed by t-test, and count data were presented as a percentage. Comparison between groups was performed using the $\chi^{2}$ test. $\mathrm{P}<0.05$ was considered to indicate a statistically significant difference.

\section{Results}

Comparison of the Barthel index, average total score of patient satisfaction with nursing care and the length of hospital stay in the two groups. Results from the comparative studies on the Barthel index scores for the two groups prior to nursing, did not reveal any significant differences $(\mathrm{P}>0.05)$. The Barthel index scores in the two groups were compared following nursing care, we observed generally higher scores for the two groups. However, patients in the observation group registered significantly higher scores as compared to the control group. Differences were statistically significant $(\mathrm{P}<0.05)$. For the observation group we also obtained significantly higher post-nursing satisfaction rate scores, and significantly shorter length of hospital stay. Differences were statistically significant $(\mathrm{P}<0.05$; Table I).

Comparison of incidence of complications in the two groups. The incidence of complications (fever, infection, bedsore, gastrointestinal function and electrolyte disturbances, and malnutrition) for our observation group was significantly lower than that the control group. The differences were significant $(\mathrm{P}<0.05$; Table II).

Comparison of FIM and Fugl-Meyer scores in two groups after 6 months follow up. Prior to nursing care, FIM and Fugl-Meyer scores did not exhibit any significant differences between the two groups ( $\mathrm{P}>0.05)$. However, FIM and Fugl-Meyer scores in the two groups after 6 months follow up registered an increase. The scores for the observation group were significantly higher than those for the control group and the differences were statistically significant $(\mathrm{P}<0.05$; Table III).

\section{Discussion}

Acute cerebral hemorrhage has been linked to hypertension, atherosclerosis, coronary heart disease, diabetes and other chronic diseases (1). Scientific and effective nursing with active and accurate clinical treatment is considered essential for improving the prognosis as well as the cure rate.

The traditional nursing mode has been shown to consist of a few flaws and due to its backward-leaning concept and the one-size-fits-all way of thinking, this method is not completely adjusted to the progression of the disease (9). Therefore, the traditional nursing mode is not ideal in attending to some of the most crucial clinical needs of the patients. The clinical nursing path is a nursing plan specialized for nursing and medical staff. This plan may be implemented on certain types of patients. This path intends to greatly ameliorate the quality of nursing by giving patients a central role while being surrounded by nursing and medical staff to offer their best efforts in order to satisfy patient needs (10). This method has been validated by cross health professionals, special disease design-associated treatment, examination, treatment and ancillary care programs (11). This mode can be presented in tabular form, aiding patients to understand self-care program content and the implication of active participation in disease rehabilitation (12).

The clinical nursing pathway includes the formulation and implementation steps. In a preceding study (13), the nursing path was formulated by a specialized nursing team, including the level above the attending physician, nurses and head nurse. The main reference was domestic and foreign-associated studies and the specific circumstances of the patients in the selected group. In the process of implementation of the nursing path, it should be carried out in strict accordance with the formulated plan. Subsequently, the finished part should be indicated, while the unfinished part constitutes the main content of the following phase of nursing (14). Supervision and evaluation of nursing care was an important aspect of the implementation of the nursing path. The head nurse and primary nurse evaluated the process and results of nursing care, which greatly promoted the improvement of nursing quality (15). The main content of the nursing path in this study included admission and discharge guidance, health education and dietary guidance. As mentioned earlier, the clinical path had obvious advantages, including the diagnosed nursing process in the emergency triage prior to admission, and the specialized procedure subsequent to the acute cerebral hemorrhage. By creating a model of mutual interaction between the nurses and patients, this method provided the patient with a central role and by combining the nursing theory with the specific situation of the patient, the patients were actively involved in the process of nursing. Additionally, this method had the potential to effectively reduce nursing management costs, and greatly reduce the waste of resources in hospital care (16). Our study revealed that implementation of this method improved the clinical outcome effect, improved the patient satisfaction rate, and reduced the length of hospital stay. We also observed that subsequent to nursing care, the Barthel index scores in our observation group were significantly higher than those of the control group. The average patient satisfaction scores on hospital nursing care were significantly higher in the observation group. Additionally, the length of hospital stay and incidence of complications for patients in our observation group was significantly lower and the differences were statistically significant. The FIM and Fugl-Meyer scores after 6 months follow up in the observation group were significantly higher than the FIM and Fugl-Meyer scores in the control group, and the differences were statistically significant. Thus, this method reduced the complications during the development period of the disease, and improved the mid-term prognosis.

In summary, the results have shown that application of the clinical nursing path for patients suffering from acute cerebral hemorrhage can significantly improve short- and long-term 
clinical effects of the treatment while improving nursing satisfaction, and reducing adverse reactions.

\section{References}

1. Manning LS and Robinson TG: New insights into blood pressure control for intracerebral haemorrhage. Front Neurol Neurosci 7: $35-50,2015$

2. Sato S, Carcel C and Anderson CS: Blood pressure management after intracerebral hemorrhage. Curr Treat Options Neurol 17: 49, 2015.

3. Yousef KM, Balzer JR, Bender CM, Hoffman LA, Poloyac SM, Ye F and Sherwood PR: Cerebral perfusion pressure and delayed cerebral ischemia after aneurysmal subarachnoid hemorrhage. Am J Crit Care 24: e65-e71, 2015.

4. Christensen MC, Mayer S and Ferran JM: Quality of life after intracerebral hemorrhage: results of the Factor Seven for Acute Hemorrhagic Stroke (FAST) trial. Stroke 40: 1677-1682, 2009.

5. Loeb M, Carusone SC, Goeree R, Walter SD, Brazil K, Krueger P, Simor A, Moss L and Marrie T: Effect of a clinical pathway to reduce hospitalizations in nursing home residents with pneumonia: a randomized controlled trial. JAMA 295 2503-2510, 2006.

6. Waguchi H, Hidaka K, Shinoki K, Matsuoka M, Mito S, Doi S, Hata A, Ibata T, Komuro R and Iijima S: Coordination to assist with hospital-to-home discharge after cerebral hemorrhage - perspectives of a patient's family and nurse. Gan To Kagaku Ryoho 40 (Suppl 2): 216-218, 2013 (In Japanese).

7. López Díaz C: Neurologically critical patient. Nurses' care. Rev Enferm 32: 49-58, 2009 (In Spanish).
8. Presciutti M: Nursing priorities in caring for patients with intracerebral hemorrhage. J Neurosci Nurs 38 (Suppl 4): 296-299, 315, 2006.

9. Waguchi H, Hidaka K, Shinoki K, Matsuoka M, Mito S, Doi S, Hata A, Ibata T, Komuro R and Iijima S: Coordination to assist with hospital-to-home discharge after cerebral hemorrhageperspectives of a patient's family and nurse. Gan To Kagaku Ryoho 40: 216-218, 2013 (In Japanese).

10. Josephson L: Management of increased intracranial pressure: a primer for the non-neuro critical care nurse. Dimens Crit Care Nurs 23: 194-207, 2004.

11. Presciutti M: Nursing priorities in caring for patients with intracerebral hemorrhage. J Neurosci Nurs 38: 296-299, 315, 2006.

12. Minier D, Milan C, Woimant F, Bematru I, Fournier O, Osseby GV, Saillard B and Giroud M: Evaluation of care given by nurses during the acute stage of stroke. Rev Neurol (Paris) 160: 1040-1047, 2004 (In French).

13. Luck L, Wilkes L and O'Baugh J: Treading the clinical pathway: A qualitative study of advanced practice nurses in a local health district in Australia. BMC Nurs 14: 52, 2015.

14. Ozasa Y: A study on first-time stroke patients in a community use of home visiting assessment by public health nurses (PHNS). Nihon Koshu Eisei Zasshi 49: 1076-1086, 2002 (In Japanese).

15. Han KT, Kim SJ, Jang SI, Kim SJ, Lee SY, Lee HJ and Park EC Positive correlation between care given by specialists and registered nurses and improved outcomes for stroke patients. J Neurol Sci 353: 137-142, 2015.

16. Pierce LL, Steiner V, de Dios AM, Vollmer M, Govoni AL and Thompson TL: Qualitative analysis of a nurse's responses to stroke caregivers on a web-based supportive intervention. Top Stroke Rehabil 22: 152-159, 2015. 